\title{
Mass Culturing of Stem and Bulb Nematode (Ditylenchus dipsaci) for use in screenİng and İmpression Training on Carrot Discs
}

\author{
Tohid Behmand ${ }^{1}$, Lerzan Ozturk ${ }^{2}$, İbrahim Halil Elekcioğlu ${ }^{1}$ \\ ${ }^{I}$ Çukurova University, Faculty of Agriculture, Department of Plant Protection, 01360, Balcall, Adana, Turkey, \\ ${ }^{2}$ Viticulture Research Institute ,Tekirdag, Turkey
}

\begin{abstract}
Stem and bulb nematode, Ditylenchus dipsaci, is a very important plant parasitic nematode, has a very wide host range and causes economic yield losses in many cultural plants in worldwide. Its races are very diverse and found in most temperate areas of the world. D. dipsaci is a migratory endoparasite, has the ability to enter into a dormancy stage. Genetic resistance offers one of the best control methods within the integrated pest management strategies for D. dipsaci. However mass rearing of stem and bulb nematode requires for the resistant studies including screening for this nematode species. The aim of study is focused on alternative rearing methods using carrot discs as a food source, culturing medium and provide a clearly outlined and visually informative guide. Carrot discs enable the rearing of high numbers of individuals of D. dipsaci for timely use in experiments and for screening purposes in under sterile conditions to provide a clean, same and pure source of inoculum. The carrot disc method has been shown to be suitable for stem and bulb nematode multiplication.
\end{abstract}

Keyword-Resistant,stem and bulb nematode,culture,Technique.

\section{SUMMARY}

Nematologists have a long time were looking to find the best methods for culture and increase population density of plant parasitic nematodes (5)., but these techniques generally has not easy. Although,the three most commenly used techniques for culturing migratory endoparasitic nematodes, including alfa alfa callus $(1,3,6)$,root expland (4), and carrot discs (5) have been published. We present in this study tried to use carrot cultur method to increase population density of nematodes. Ditylenchus dipsaci or stem nematode, attacks more than 1200 type of wild and cultivated plants.many agriculture plants are hosts for this species of nematode.D.dipsaci live mostly as a migratory endoparasite in different parts of plants like stem,leaves and flowers.In vitro rearing of Ditylenchusdipsaci nematodes on carrot discs was used for grown nematodes. This technique is workable to migratory nematodes (Ditylenchusspp) for serial manufacture of nematode for experimental aim, direct studies of nematodes, screening trial and increase nematodes. The most advantage this method to save timethat,allow a quick mass production of nematode. Plant parasitic nematode can only develop in fresh root tissue this method help Ditylenchusdipsaci nematode to be produce on sterilised carrot discs in petri dishes in an incubator more than in root plant under laboratory condition. Nematodes feeding two the type of plant tissue that necessary for their culture their can feed as migratory endoparasites and ectoparasites.Sedentary endoparasites nematode need individuate tissue for reproduction but it is the opposite about migratory endoparasites nematodes like Ditylenchusdipsaci that do not require for it and reproduce easily on undifferentiated tissue like as carrot disk and it has been indicated to be suitable tissue for their multiplication.This technique provides a basic facility for migratory endoparasites nematodes to be reared carrot discs in petri dishes inside an incubator more than in root of plant under greenhouse condition (8).Also, This study provides a protocol for the use carrot to produce and extract stem and bulb nematodes from carrot disk culture.

\section{MATERIAL AND METHOD}

We choose infected roots or soil with Ditylenchusdipsaci for extraction of the nematode to be culture.Nematodes were inoculated on the carrot disc.The suggested temperature for rapid multiplication between $19^{\circ} \mathrm{c}$ and $23^{\circ} \mathrm{c}$ depending on nematode reproduction population must be sub culture every 4-6 weeks, the development rate decreased when Temperatureincreased up to $30^{\circ}$ or decrease down to $18{ }^{\circ} \mathrm{c}$. The first to start a carrot culture, weselected clean carrots without overly thick and cracks, Washes selected carrots under distilled water, sterilise all of equipment and materials by autoclaving at $121^{\circ} \mathrm{c}$ for 20 
minutes and sterilise the workingand tools surface with $96 \%$ ethanol and hold the carrot with $96 \%$ ethanol and flame over spirit lamp repeat this for three times.Also, westerilised all of the equipment including: forceps,peeler and knife with ethanol and flaming eachbefore used them every time. We peeled the carrot with the sterilised peeler and Cut the peeled carrot into $5 \mathrm{~mm}$ thick section of $3-4 \mathrm{~cm}$ diameter and transfer the cut carrot disc into sterilise petri dishes by using the sterilised forceps. Disk must not be less than $3 \mathrm{~cm}$ diameter, then transfer the carrot discs into sterilise glass petri dishes $(5-6 \mathrm{~cm}$ diameter). We stored them in the dark incubator to keep underground condition for 3-4 weeks at $19-23^{\circ} \mathrm{c}$.Also,it was necessary control carrot discs best signal of healthy cultures during incubation every week. After 3-4 weeks nematodes were started to exit the carrot and when nutrient in carrots go to become empty it was indicator for harvesting the nematodes. The other words, incubation time and the initial inoculum effected how soon the nutrients become empty. For selection of nematodes we placed a concentrated of the nematode extract into a small (3-5 cm diam.) andaccounted under light microscope.The number of nematode for inoculation depends how many carrots are to be required. However,it was suggested to inoculate at least 100-150 nematodes on one carrot disc and not inoculate less than 100 of them.It was recommended to inoculate at least five replicates from petri dishes .For mass multiplication we increasednumber of inoculated nematode on one carrot disc and selected female and male of the Ditylenchusdipsaci nematode and placed them in a glass petri including sterile distilled water.The aim was to deliver 50-80 nematodes per disc in a maximum of two 50 microliter smaller drops of nematode suspension transfer the nematodes. The number of nematodes in the suspension were determined the number to transfer. It was suggested use about 80-150 nematodes for mass multiplication. After that, we placed the carrot disk in an incubator for 5-6 week, after thanthe nematodes were harvested and stored in the fridge at $5^{\circ} \mathrm{c}$,the nematode can remain viable for up to 1-2 week. For a more completed collection, the first cut carrot disc to small pieces and placed in the petri dishes with sterile water and let the nematodes migrate freely into the petri, thenextracted nematodes counted under binocular microscope and placed in the flask.

\section{RESULT}

The number of nematodes extracted from the 45,60,90 and 120 days callus culture are listed (Table 1). 2 or 3 thousands of nematodes can be extracted out of one carrot discs after 45-60 days of culture. However, the number of nematode is different use of nematode species and geographical population The greatest number of nematodes was recoverd from the 45 old day with temperature of $19^{\circ} \mathrm{c}$ to $23^{\circ} \mathrm{c}$, which yielded a greater than 1,2 and 3 fold in number of nematodes that was observed from the 60,90 and 120 days with same temperature. The lowest number of nematodes recovery was from the 120 days with $25^{\circ} \mathrm{c}$ to $30^{\circ} \mathrm{c}$ in the carrot disk taht was only slightly increased from the initial inoculum levels. The number of nematodes extracted from callus after 45 days with $19^{\circ} \mathrm{c}$ to $23^{\circ} \mathrm{c}$ showed potential yields using this technique for us (Table 1).The other word,extracted of more than 3400 nematodes were observed formthe 45 days with $19^{\circ} \mathrm{c}$ to $23^{\circ} \mathrm{c}$. Therfore, temperature between $19{ }^{\circ} \mathrm{C}$ to $23^{\circ} \mathrm{C}$ could increase the cultivation potential of Ditylenchus dipsaci nematode.Also, These result indicated that, when as the number of days increased and the temperature dropped under $19^{\circ} \mathrm{c}$ or increased up to $23^{\circ} \mathrm{c}$, the number of nematodes decreased.Becuace of that we observedthat The stem and bulb (ditylenchus dipsaci) nematode was suited for culture carrot disk and the yield of eggs and increased population density of this migratory endoparasite nematode was much better by this technique than other extraction and culture methodes $(2,5,7,8,9)$.Therfore, the carrot callus method was suitable and easy to use and produce high number of Ditylenchus dipsaci(Fig 1).

Table.1: The effect of different days and temperature on the development of the Ditylenchus dipsaci nematode.

\begin{tabular}{|c|c|c|c|}
\hline Nematode number & $15-18^{\circ} \mathrm{c}$ & $19-23^{\circ} \mathrm{c}$ & $25-30^{\circ} \mathrm{c}$ \\
\hline 45 day-old cultur & 2200 & 3400 & 1400 \\
\hline 60 day-old culture & 2000 & 3000 & 1100 \\
\hline 90 day-old culture & 1100 & 1400 & 900 \\
\hline 120 day-old culture & 600 & 800 & 500 \\
\hline
\end{tabular}




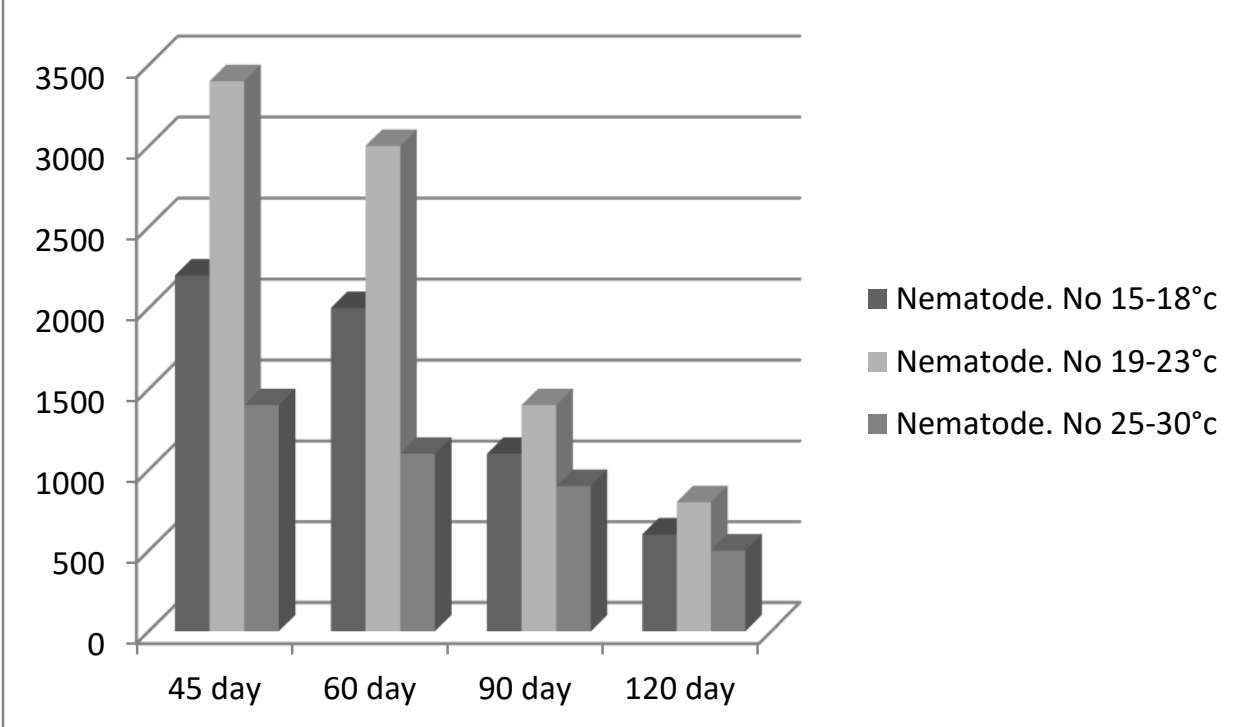

Fig.1: Mixed life stage and eggs number ofDitylenchus dipsaci nematode from 45,60,90 and 120 days old carrot callus culture.

\section{DISCUSSION}

This protocol presented is now routinly used in our laboratory.The condition of carrots was importent, work (7) has shown that carrots used in carrot disk culture must be freshly harvested from the field and greenery intact before use.Also, carrots that are badly cut must be avoided.Culture can remain available for up to 2 months and are easly generated.Once the nematode are established on the callus can be divided to small infested piece and transferred directly to make a new carrot culture. The yield of eggs and increased population density of this migratory endoparasite nematode was much better by this technique than other extraction and culture methodes $(2,5,7,8,9)$.

\section{REFERENCES}

[1] Bingefors, S., and K. B. Eriksson. 1963. Rearing stem nematode inoculum on tissue culture: Preliminary report. Landbrukshogskolan Annaler 29: 107-118.

[2] Huettel, R. N. 1985. Carrot disc culture. Pp. 212 in B. M. Zuckerman, W. F. Mai, and M. B. Harrison, eds. Plant nematology, laboratory manual. University of Massachusetts Agricultural Experiment Station, University of Massachusetts, Amherst.

[3] Krusberg, L. R. 1961. Studies on the culturing and parasitism of plant-parasitic nematodes, in particular Ditylenchus dipsaci and Aphelenchoides ritzemabosi on alfalfa tissues. Nematologica 6:181-200.

[4] Lauritis, J. A., R. V. Rebois, and L. I. Graney. 1982. Gnotobiotic cultivation of Heterodera glycines Ichinohe on Glycine max (L.) Merr. Journal of Nematology 14:422-424..
[5] Lawn, D. A., and G. R. Noel. 1984. Monoxenic culture of Pratylenchus scribneri on carrot disks. Proceedings of the First International Congress of Nematology. P. 47 (Abstr.)

[6] Myers, R. J., W. A. Feder, and P. C. Hutchens. 1965. The rearing ofRadopholus similis (Cobb) Thorne on grapefruit, okra, and alfalfa root callus tissues. Proceedings of the Helminthological Society of Washington 32:94-95

[7] Moody, E. H., B. F. Lownsbery, and J. M. Ahmed. 1973. Culture of the root-lesion nematode Pratylenchus vulnus on carrot disks. Journal of Nematology 5:225-226.

[8] O'Bannon,J. H., and A. L. Taylor. 1968. Migratory endoparasitic nematodes reared on carrot discs. Phytopathology 58:385.

[9] Young, T. W. 1954. An incubation method for collecting migratory endo-parasitic nematodes. Plant Disease Reporter 38:794-795 